\title{
Evidence for a dissociation between the control of oculomotor capture and disengagement
}

\author{
Sabine Born · Dirk Kerzel · Jan Theeuwes
}

Received: 13 April 2010 / Accepted: 23 November 2010 / Published online: 25 December 2010

(C) The Author(s) 2010. This article is published with open access at Springerlink.com

\begin{abstract}
The current study investigated whether capture of the eyes by a salient onset distractor and the disengagement of the eyes from that distractor are driven by the same or by different underlying control modes. A variant of the classic oculomotor capture task was used. Observers had to make a saccade to the only gray circle among red background circles. On some trials, a green (novel color), red (placeholder color) or gray (target color) distractor square was presented with sudden onset. Results showed that when participants reacted fast, oculomotor capture was primarily driven by bottom-up pop-out: both types of distractors (green and gray) that popped out among the red background elements showed more capture than a red distractor that did not pop-out. In contrast to initial capture, disengagement of the eyes from the distractor was driven by top-down target-distractor similarity effects. We also examined the time-course of this effect. The distractor could change from green to either the target or placeholder color. When the color change was early in time (30-40 ms after its onset), dwell times were strongly affected by the change, whereas the effect on oculomotor capture was weak. Importantly, a change occurring as early as 60-80 ms after distractor onset did neither affect capture nor dwell times, corroborating the assumption of parallel programming of saccades.
\end{abstract}

S. Born $(\varangle) \cdot$ D. Kerzel

Faculté de Psychologie et des Sciences de l'Éducation,

Université de Genève, 40 Boulevard du Pont d'Arve,

1205 Genève, Switzerland

e-mail: sabine.born@unige.ch

J. Theeuwes

Department of Cognitive Psychology,

Vrije Universiteit Amsterdam,

Van der Boechorststraat 1, 1081 BT Amsterdam, The Netherlands
Keywords Oculomotor capture · Disengagement . Bottom-up $\cdot$ Top-down $\cdot$ Parallel programming of saccades

\section{Introduction}

While searching for a pre-defined target stimulus, irrelevant visual events may involuntarily capture our gaze (e.g. Theeuwes et al. 1998, 1999, 2003; Irwin et al. 2000; Wu and Remington 2003; Ludwig et al. 2008). The phenomenon has been termed oculomotor capture. However, after being captured, our gaze dwells only for a short period of time on a distractor stimulus before it can be disengaged again, and a subsequent corrective saccade toward the target is initiated (Theeuwes et al. 1999; Godijn and Theeuwes 2002; Mulckhuyse et al. 2008).

Even though oculomotor capture and subsequent oculomotor disengagement from the distractor have been well described in the literature (e.g. Godijn and Theeuwes 2002; Mulckhuyse et al. 2008), one important question that has not been answered yet is whether oculomotor capture and oculomotor disengagement are driven by the same underlying control modes (bottom-up vs. top-down): Is the control of capture and disengagement closely coupled, for instance, does bottom-up capture on a given trial result in oculomotor disengagement being likewise controlled by bottom-up factors? Oculomotor capture has been explained by a competitive process between two activated sites (corresponding to target and distractor location) in a retinotopically organized saccade motor map (e.g. Findlay and Walker 1999; Trappenberg et al. 2001; Godijn and Theeuwes 2002). Depending on the site that wins the competition, the saccade may be either directed toward the target or toward the distractor. The occurrence of capture may thus reflect the strength of the competition signal evoked by the distractor 
when compared to the target signal. Along these lines, it has been found that luminance increments are less efficient than onsets in capturing our gaze (Irwin et al. 2000). Further, color singleton distractors may not capture the eyes when observers are asked to saccade toward an onset target; however, they may produce oculomotor capture when observers are looking for a shape singleton target (Irwin et al. 2000; Theeuwes et al. 2003).

It is less clear how oculomotor disengagement from the distractor is controlled. Typically, the eyes only dwell for about $100 \mathrm{~ms}$ on the distractor before they are disengaged. This is shorter than one would expect the full programming of a visually guided saccade to take (around 150-200 ms). Therefore, it has been argued that the corrective saccade is partly prepared in parallel with the initial capture saccade (e.g. Theeuwes et al. 1999; McPeek et al. 2000; Godijn and Theeuwes 2002). It is assumed that target-related activity in the saccade map can be maintained across the capture saccade, allowing for the initiation of a fast corrective saccade. Neurophysiological data support this assumption (McPeek and Keller 2002; Murthy et al. 2007). However, less thought has been given to the issue what happens to the distractor-related signal in the saccade map, once it has won the competition (and subsequently elicited a capture saccade). Some studies have shown that a distractor that shares some relevant features with the target does not only produce more oculomotor capture than a distractor that is dissimilar from the target; a target-similar distractor also provokes longer gaze dwell times before the eyes can be disengaged from it (Ludwig and Gilchrist 2002, 2003a, b; Mulckhuyse et al. 2008, 2009; Becker et al. 2009). This suggests that similar to the target-related signal, distractorrelated activity may also linger in the oculomotor system, even after it has won the competition for saccade initiation. To execute the corrective saccade, the residual distractorrelated activity has to be overcome. This should be more time-consuming if the signal was stronger in the first place and consequently, gaze dwell times on the distractor should be longer before the eyes can disengage. Accordingly, distractors that prompt more oculomotor capture should also produce longer gaze dwell times on the distractor. In other words, according to this view, the control of capture and disengagement should be closely coupled.

However, the above-mentioned results do not exclude a dissociation in the control of capture and disengagement. In particular, oculomotor capture can be envisaged to be more strongly driven by bottom-up factors. Per definition, a capture saccade is an erroneous response, that is, a premature response that is initiated before all relevant (top-down) information that distinguishes between target and distractor has reached the oculomotor system. This is also evident in the finding that capture saccades are usually initiated earlier than regular target-directed saccades (e.g. Theeuwes et al.
1999; Godijn and Theeuwes 2002; Ludwig and Gilchrist 2002; Mulckhuyse et al. 2008, 2009). In contrast, how fast the eyes can disengage from an erroneously fixated stimulus may always have to rely on top-down control. The initiation of a corrective saccade depends critically on participants monitoring their errors, that is, on the top-down identification and rejection of the fixated stimulus as distractor and/or the localization of the target somewhere else in the visual field. That such processes take place can be easily seen in the fact that capture saccades are almost always followed by a subsequent corrective saccade in the oculomotor capture paradigm, whereas correct targetdirected saccades entail almost never a subsequent distractordirected saccade. The ease and speed of distractor rejection as saccade target may critically depend (among other factors) on target-distractor similarity. In other words, error monitoring should be easier when the distractor is of a different color than the target and should be more difficult and time-consuming when the distractor is of the same color as the target. In sum, distractors sharing target features may produce more oculomotor capture because they produce a stronger distractor signal than dissimilar distractors. However, they may provoke longer gaze dwell times for a different reason: because of their similarity to the target that makes error monitoring more difficult and timeconsuming.

The goal of the current experiment was to test whether effects on oculomotor disengagement can be decoupled from the initial capture effect. We used a variant of the classic oculomotor capture task (Theeuwes et al. 1998, 1999; see Fig. 1) and deliberately created conditions that favored stimulus-driven capture by using sudden onset stimuli and a gap procedure. Sudden onset distractors are generally found to be very efficient in stimulus-driven capture of attention and the eyes (e.g. Todd and Gelder 1979; Jonides 1981; Yantis and Jonides 1984; Yantis 1993; Theeuwes 1994; Irwin et al. 2000). A gap procedure, that is, the offset of the fixation cross prior to the onset of the target display has proven to be successful in reducing saccade latencies in oculomotor tasks (e.g. Saslow 1967; Reuter-Lorenz et al. 1991; Kingstone and Klein 1993; McSorley et al. 2006; Mulckhuyse et al. 2009). It has been suggested that shortlatency saccades show more stimulus-driven capture than long-latency saccades (Godijn and Theeuwes 2002; Ludwig and Gilchrist 2002; Mulckhuyse et al. 2008). We compared the impact of three distractor types on capture: a distractor presented in the target color that popped out among the surrounding stimuli (i.e. the distractor stood out due to its local color contrast), a distractor presented in the color of the surrounding stimuli (different from the target, no pop-out) and a distractor presented in a novel color (different from the target and from the surrounding stimuli, pop-out). For stimulus-driven capture, we expected the two 


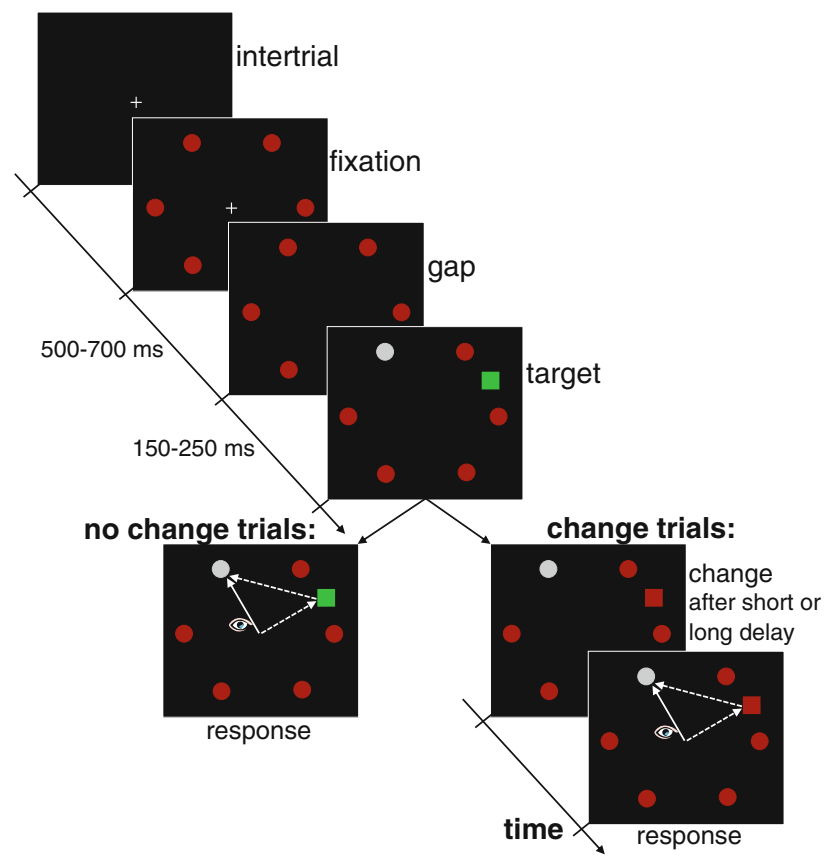

Fig. 1 Sequence of events. Participants initiated the trial by pressing the space bar. Six red placeholder circles appeared on screen. After a random interval of 500-700 ms, the fixation cross was extinguished. Following a $150-250 \mathrm{~ms}$ gap period, one of the placeholders changed to gray (target color change). Participants' task was to make an eye movement toward the gray circle as fast as possible. On no change trials, simultaneously with the target, a green, gray or red distractor square could be presented at a previously empty location. On change trials, the distractor was initially presented in green, but changed subsequently to gray (target color) or red (placeholder color; as shown in the figure) either after a short delay $(30 / 40 \mathrm{~ms})$ or a slightly longer delay $(60 / 80 \mathrm{~ms})$. Arrows in the last panels indicate that participants responded either with a correct saccade toward the target (straight line) or with a capture saccade toward the distractor, followed by a corrective saccade toward the target (dotted lines)

pop-out distractors to provoke more capture than the distractor that is presented in the color of the surrounding stimuli, irrespective of whether the pop-out distractor color is the same or different from the target color. We may then look at dwell times and compare whether the same pattern of results is found or whether the dwell time pattern suggests top-down influences, that is, longer dwell times on the distractor that is presented in the target color and shorter dwell times on the other distractors, irrespective of whether they pop-out among the surrounding stimuli.

Additionally, we made a first attempt to examine up to which moment in time oculomotor capture and disengagement could be influenced. To this end, we included trials where the distractor square was initially presented in the novel color. Subsequently, the distractor could change to either the target color or to the color of the surrounding stimuli at various delays after its onset. The rationale behind this manipulation is reminiscent of the classic double-step paradigm (Becker and Jürgens 1979). In this task, participants are asked to track with their eyes a visual target that makes one or two unpredictable steps on every trial. On double-step trials, if the second target step occurs well before the first eye movement can be initiated, participants will only make one single saccade directed to the final target location. However, if the second target step occurs a little later in time, that is, shortly before the first saccade is initiated, participants follow the target in two saccades, mimicking the target displacement. Apparently, saccade programming of the first saccade has already progressed up to a point where new information about the second target step cannot be considered anymore. In the current study, we were not only interested in when the first eye movement in a series of saccades cannot be affected anymore, but also when the parameters of the corrective saccade are set. To this end, we used two fixed delays at which the distractor could change color to coarsely examine from which moment in time the color change in the distractor could not influence dwell times anymore.

\section{Methods}

\section{Participants}

Ten students ( 7 women) from the Vrije Universiteit Amsterdam participated in the experiment. Observers ranged from $18-25$ years of age and had normal or corrected to normal vision.

\section{Apparatus}

The experiment was run on a Pentium IV computer with a processor speed of $3 \mathrm{GHz}$. Stimuli were presented on an Iiyama 21" SVGA monitor with a resolution of 1024 pixels $\times 768$ pixels and a refresh rate of $100 \mathrm{~Hz}$. The displays were generated using the E-Prime 2.0 application suite (Psychology Software Tools, Inc., Pittsburgh, PA). A second computer controlled the registration of eye movement data online. Eye movements were recorded by means of an EyeLink1000 video-based eye tracker at a sampling rate of $1,000 \mathrm{~Hz}$ (SR Research Ltd, Canada). Only the right eye was monitored, and the participant's head was stabilized by a chin rest. The experiment was conducted in a dimly lit room, and participants were seated at a distance of $72 \mathrm{~cm}$ from the screen. For detection of fixations, saccades and blinks, the automatic detection algorithms of the EyeLink system were used.

\section{Stimuli}

Stimuli were presented on a black background $\left(\sim 0.2 \mathrm{~cd} / \mathrm{m}^{2}\right)$. Six red circles $\left(1.5^{\circ}\right.$ in diameter) were presented at $1,3,5$,

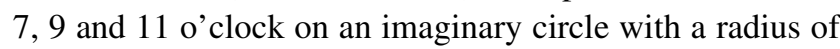
$9.5^{\circ}$ of visual angle. The target color change to gray could 
only take place at the 1-, 5-, 7- and 11-o'clock positions. The distractor square $\left(1.5^{\circ}\right.$ side length) could be presented at 2, 4, 6 or 10 o'clock on the same imaginary circle but always with an angular distance of at least $90^{\circ}$ to the gray target. Gray $(x=0.29, y=0.33)$, red $(x=0.63, y=0.33)$ and green $(x=0.30, y=0.60)$ stimuli were matched for luminance $\left(10,10\right.$ and $11 \mathrm{~cd} / \mathrm{m}^{2}$, respectively).

\section{Procedure}

The sequence of events is illustrated in Fig. 1. At the beginning of each trial, participants were asked to fixate a central white cross and then to press the space bar on a common PC keyboard to launch the stimulus presentation. First, the six red placeholder circles appeared on screen. After a random interval of 500-700 ms, the fixation cross was extinguished. A random gap period of $150-250 \mathrm{~ms}$ followed, and then a color change to gray in one of the circles defined the target for the saccadic response. On $87.5 \%$ of trials, a distractor square was presented with a sudden onset and at the same time as the target circle changed to gray. Participants were informed that the square was irrelevant to the task and that they should try to ignore it. They were asked to fully concentrate on the gray circle and to react as fast as possible. The distractor square was presented in green (novel color, pop-out), gray (target color, pop-out) or red (placeholder color, no pop-out). The distractor color stayed either constant until the end of the trial (no change trials). Or, if presented in green, the distractor could change to the target or the placeholder color either after a short delay or after a slightly longer delay. As the exact time-course of the underlying processes was unclear, we tested 5 participants in a condition with a 30 -ms short delay and a $60-\mathrm{ms}$ long delay, and 5 participants with a 40-ms short and a 80-ms long delay. Statistical analyses showed no differences between the two groups; therefore, the data were collapsed in the reported results. Note that in both delay conditions, the distractor changed color well before the first saccade could be initiated. This procedure was chosen as previous studies already demonstrated that changes occurring during the first saccade do not influence the second saccade anymore (McPeek et al. 2000; Godijn and Theeuwes 2002). Finally, 1,000 ms after target onset, the stimulus display was extinguished, and the fixation cross came up again to signal the beginning of the next trial. Additionally, two possible feedback messages could appear for $1,000 \mathrm{~ms}$ at the screen center at the end of a trial: "fixation error" was shown if participants' gaze drifted more than $1^{\circ}$ away from the screen center during the fixation or the gap period; "too slow" was shown if participants' gaze had remained within $2^{\circ}$ of the screen center during the first $350 \mathrm{~ms}$ after target onset (i.e., no saccade had been made).
Design

All conditions were presented with equal probability $(12.5 \%)$ and were randomly interleaved. The experiment consisted of 1 practice block of 20 trials and 10 experimental blocks of 96 trials run in a single 90-min session with several breaks.

\section{Results}

We excluded trials with saccadic reaction times shorter than $80 \mathrm{~ms}$ as anticipations and trials that were marked with an error message as "fixation error" or as "too slow". Further, we removed trials when a blink was detected in the interval from fixation onset until the eyes had landed on the target. We also excluded trials with redirected saccades. On those trials, the first saccade showed a trajectory that was initially directed toward the distractor. However, it never landed on the distractor but turned around in midflight to land on the target. As these trials could not clearly be classified as capture or correct saccades, we excluded them from analysis. In total, around $4 \%$ of trials were removed due to the occurrence of redirected saccades. Finally, we only considered trials in which the first saccade went either in the direction of the target (i.e. saccade endpoint less than 45 angular degree away from the target) or toward the distractor (with a subsequent saccade to the target). In total, $14.8 \%$ of trials were discarded from analysis.

Trials without distractor color change

By means of a repeated-measures ANOVA with distractor color as factor, we first analyzed the percentage of capture in the conditions in which the distractor did not change color but was presented in the novel, target or placeholder color and stayed that way throughout the trial. The main effect was significant, $F(2,18)=31.15, p<.001$, revealing that there were differences between distractor color conditions. To see whether capture was influenced by bottom-up color pop-out effects or by top-down target-distractor similarity effects, we ran subsequent pairwise $t$ tests. Bottom-up color pop-out should result in a significant difference between the novel color and the placeholder color condition: both are dissimilar from the target, but only the novel color distractor pops out among the placeholders. Indeed, there was a significant difference between the two conditions, $t(9)=7.22, p<.001$. Figure $2 \mathrm{a}$ illustrates that the proportion of capture saccades was higher for the novel color distractor. In contrast, top-down similarity effects should produce a significantly larger proportion of capture for the target color distractor than the novel color distractor: both show pop-out, but only the target color distractor is 
Fig. 2 Percentage of capture saccades (a), gaze dwell times on the distractor (b) and saccadic latencies for capture saccades (c) in the no change conditions. The bar color represents the color of the onset distractor. Error bars represent within-subjects 95\% confidence intervals (see Bakeman and McArthur 1996)
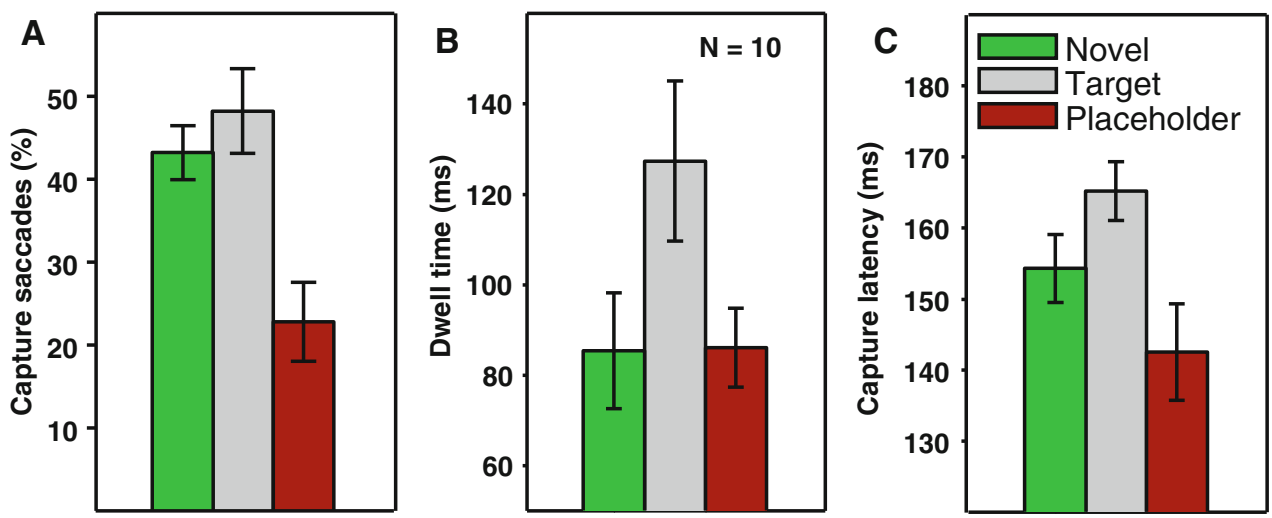

similar to the target. However, this difference did not reach significance, $t(9)=1.59, p=.147$.

The same analyses that we performed on the capture data were also run on dwell times on the distractor. Figure $2 b$ illustrates the results. A repeated-measures one-way ANOVA showed again a significant main effect of distractor color, $F(2,18)=10.66, p=.001$. However, the subsequent pairwise $t$ tests revealed a different pattern than for percentage of capture. There was no significant difference between the novel color and the placeholder color conditions, $t(9)=0.12, p=.905$, revealing a lack of a bottom-up pop-out effect. In contrast, the target-colored distractor produced significantly longer dwell times than the novel color distractor, $t(9)=3.20, p=.011$, indicating an effect of topdown target-distractor similarity.

Finally, we also looked at differences in saccadic latencies for capture saccades (Fig. 2c). A repeated-measures one-way ANOVA, $F(2,18)=15.25, p<.001$ revealed a main effect of the distractor color. Pairwise $t$ tests showed effects of both popout and similarity. Saccadic latencies were significantly longer for target color than for novel color distractors, $t(9)=4.24$, $p=.002$, which were in turn longer than the latencies for placeholder color distractors, $t(9)=2.43, p=.038$.

Effects of the distractor color change

Next, we tried to estimate up to which moment in time a color change had to occur to still have an influence on capture and disengagement. To this end, we compared the target color and the placeholder color trials across change delay conditions (no change, short delay, long delay). We first ran a 2 (distractor color) $\times 3$ (change delay) repeated-measures ANOVA on the capture data (see Fig. 3a). There were significant main effects of distractor color, $F(1,9)=22.34, p=.001$, as well as change delay, $F(2,18)=7.93, p=.003$. More importantly, there was a significant two-way interaction between the two factors, $F(2,18)=31.90, p<.001$. Subsequent pairwise $t$ tests confirmed that there was a large and significant difference in percentage of capture between the target color and the placeholder color distractors in the no change condition, $t(9)=6.16$, $p<.001$, a strongly reduced, but still significant, difference in the short delay condition, $t(9)=3.36, p=.008$, and no difference in the long delay condition, $t(9)=2.17, p=.059$. If anything, the effect in the long delay condition was reversed (i.e. more capture for placeholder color distractors than target-colored distractors). Further, the difference between target and placeholder color distractors was significantly larger in the no change condition than in the short delay condition, $t(9)=4.22$, $p=.002$, which in turn was larger than in the long delay condition, $t(9)=5.25, p=.001$.

Comparing the effect of the color change across change delay conditions in dwell times (Fig. 3b) likewise revealed significant main effects of distractor color, $F(1,9)=16.32$, $p=.003$, change delay, $F(2,18)=5.77, p=.012$, as well as a significant two-way interaction between the two factors, $F(2,18)=10.90, p=.001$. In the subsequent $t$ tests, highly significant differences between the target and placeholder color conditions were found in the no change trials, $t(9)=3.76, p=.004$, and the short delay condition, $t(9)=4.37, p=.002$, whereas no difference was found in the long delay condition, $t(9)=0.88, p=.402$. In contrast to the capture data, the difference in the no change trials was not significantly larger than in the short delay condition, $t(9)=1.06, p=.319$. However, as for capture, the difference was significantly larger in the short delay compared with the long delay condition, $t(9)=4.07, p=.033$.

Finally, the same ANOVA run on the latencies of capture saccades (Fig. 3c) showed a significant main effect of distractor color, $F(1,9)=26.05, p=.001$, but no main effect of change delay, $F(2,18)=1.57, p=.235$. However, the interaction between the two factors reached significance, $F(2,18)=8.48, p=.003$. Subsequent $t$ tests showed significant differences between target and placeholder color conditions in the no change condition, $t(9)=5.03, p=.001$ and the short delay condition, $t(9)=3.21, p=.011$, but not in the long delay condition, $t(9)=1.00, p=.334$. Furthermore, the difference was not larger in the no change condition than in the short delay condition, $t(9)=1.47, p=.177$, but the short delay condition showed a significantly larger effect than the long delay condition, $t(9)=4.04, p=.003$. 

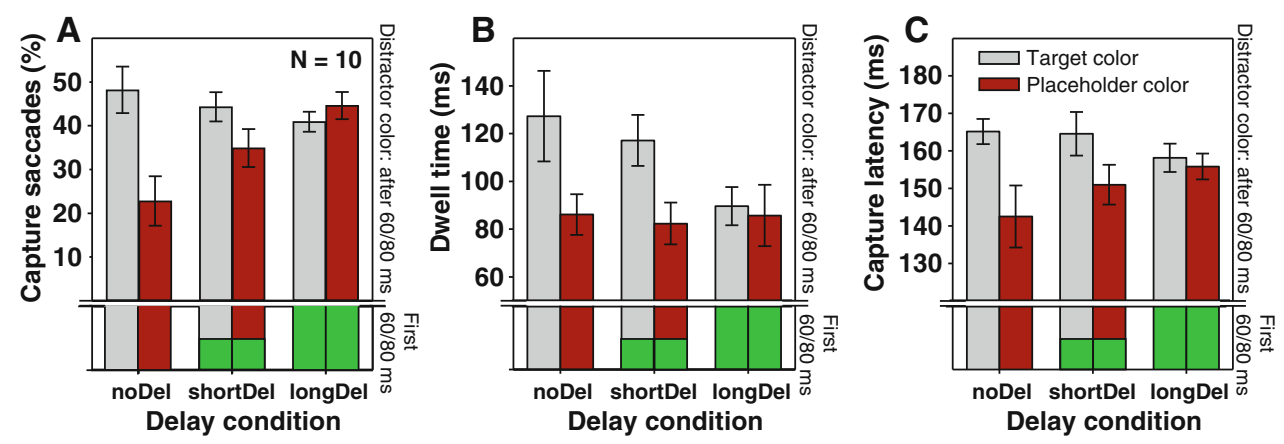

Fig. 3 Percentage of capture saccades (a), gaze dwell times on the distractor (b) and saccadic latencies for capture saccades (c) as a function of change condition. The colors above the horizontal line represent the distractor color after the first $60 / 80 \mathrm{~ms}$ of its onset. The colors at the bottom of each panel represent the distractor color during the first 60/ $80 \mathrm{~ms}$. In the no delay (noDel) condition, the distractors did not change

\section{Effects aligned to saccade onset}

Typically, measures that assess up to which moment in time saccades can be influenced do not look at the effects of changes aligned on stimulus onset but aligned on saccade onset. Therefore, we also split the data in the change conditions into groups, depending on whether the change occurred early or late before capture saccade onset and whether it occurred early or late before the onset of the corrective saccade. Overall, results were similar to the data aligned to stimulus onset. First, we performed individual median splits for every participant. On average, the split value was around $100 \mathrm{~ms}$ (range across participants: 80$130 \mathrm{~ms}$ ) when aligned to the onset of the capture saccade, meaning that in the early change condition, the change occurred at least $100 \mathrm{~ms}$ before capture saccade onset, whereas in the late change condition the capture saccade was initiated within $100 \mathrm{~ms}$ after the change. When aligned to the onset of the corrective saccade, the split value was around $230 \mathrm{~ms}$ (range across participants: 206-258 ms), meaning that in the early change condition, the change occurred at least $230 \mathrm{~ms}$ before onset of the corrective saccade. Results are illustrated in Fig. 4a. Note that overall, the percentage of capture was much higher in the late change conditions compared to the early change conditions when the change was aligned to the onset of the capture saccade, whereas the reverse pattern was found when the change was aligned to the onset of the corrective saccade. This is not surprising as splitting the data according to when the change occurred with respect to saccade onset is confounded with a split of the data into two latency groups: trials in which the change occurred early before saccade onset show inevitably longer overall latencies than trials in which the change occurred shortly before saccade initiation (as the time between change and saccade onset is part of saccade latency). Note that capture saccades were initiated with color. In the short delay condition (shortDel), the distractor was initially presented in green, but changed after $30 / 40 \mathrm{~ms}$ to the target or placeholder color. In the long delay condition (longDel), the distractor changed after $60 / 80 \mathrm{~ms}$. In all panels, error bars represent within-subjects $95 \%$ confidence intervals (see Bakeman and McArthur 1996)

shorter latency than correct target-directed saccades. In fact, for correct target-directed saccades, mean saccadic latencies were at $197 \mathrm{~ms}$ in the no distractor condition and $223 \mathrm{~ms}$ averaged over all distractor conditions. Therefore, the proportion of capture is higher in short-latency (i.e. late change) trials when aligning on capture saccade onset. Conversely, corrective saccades are usually executed later than initially correct target-directed saccades (as the corrective saccade is only initiated after an error saccade), and therefore the proportion of capture is overall higher in long-latency (i.e. early change) trials when aligning on corrective saccade onset. In any case, we were interested in differences in capture between trials in which the distractor changed to the target and placeholder color across change timing conditions. A 2 (distractor color) $\times 2$ (change condition) repeated-measures ANOVA on the capture data aligned on capture saccade onset produced a highly significant main effect of change condition, $F(1,9)=59.24$, $p<.001$, no significant effect of distractor color, $F(1,9)=$ $1.71, p=.223$, but a significant two-way interaction between distractor color and change condition, $F(1,9)=$ $7.75, p=.021$. Very similar to the short and long delay conditions in Fig. 3a, changing the distractor early before capture saccade onset (see Fig. 4a, left subpanel) resulted in a small but significant difference between the two distractor color conditions, $t(9)=2.69, p=.025$, whereas there was no difference in the late change condition, $t(9)=0.73, p=.486$. In contrast, when aligned to the onset of the corrective saccade (Fig. 4a, right subpanel), the corresponding ANOVA only revealed a significant main effect of change condition, $F(1,9)=50.14, p<.001$, but no significant main effect of distractor color, $F(1,9)=0.12$, $p=.915$, and no interaction between the two factors, $F(1,9)=2.73, p=.133$.

Comparing the results of the dwell time data across early and late change conditions with respect to capture saccade 

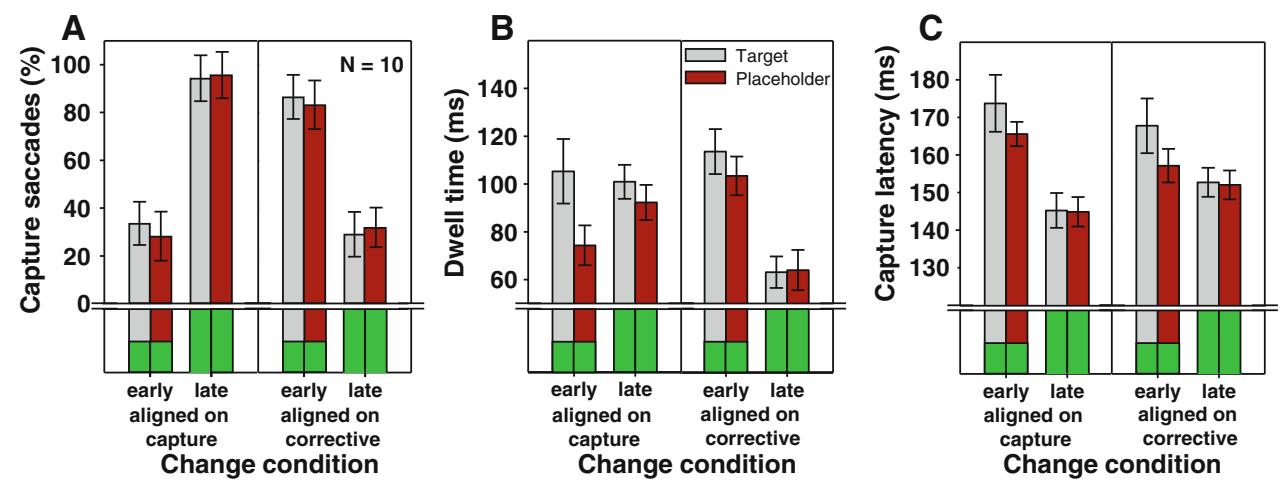

Fig. 4 Percentage of capture saccades (a), gaze dwell times on the distractor (b) and saccadic latencies for capture saccades (c) as a function of early or late change of the distractor color with respect to capture saccade onset (left subpanels) or corrective saccade onset (right subpanels). The main bar color represents the distractor color after the

onset (Fig. 4b, left subpanel) revealed a significant main effect of distractor color, $F(1,9)=14.58, p=.004$, no significant main effect of change condition, $F(1,9)=2.18$, $p=.174$, but a significant two-way interaction between the two factors, $F(1,9)=5.79, p=.039$. Subsequent $t$ tests showed significant differences between distractor color conditions when the change occurred early, $t(9)=3.52$, $p=.007$, but only a marginally significant difference when the change occurred late, $t(9)=1.95, p=.083$. When aligned on corrective saccade onset (Fig. $4 \mathrm{~b}$, right subpanel), the ANOVA produced a significant main effect of change condition, $F(1,9)=50.38, p<.001$, no significant main effect of distractor color, $F(1,9)=2.85, p=.126$, but a significant two-way interaction between the two factors, $F(1,9)=6.53, p=.031$. In the early change condition, the difference between the two distractor color conditions was significant, $t(9)=2.27, p=.049$, but not in the late change condition, $t(9)=0.44, p=.670$.

Finally, saccadic latencies across early and late change conditions with respect to capture saccade onset (Fig. 4c, left subpanel) revealed a significant main effect of change condition, $F(1,9)=52.42, p<.001$, and a marginally significant main effect of distractor color, $F(1,9)=4.56$, $p=.061$, but no significant two-way interaction between the two factors, $F(1,9)=3.18, p=.108$. When aligned on corrective saccade onset (Fig. $4 \mathrm{c}$, right subpanel), the main effects of change condition, $F(1,9)=10.36, p<.010$, and of distractor color, $F(1,9)=6.70, p=.029$, reached significance. The two-way interaction was only marginally significant, $F(1,9)=4.61, p=.060$.

\section{Discussion}

We investigated whether the capture of the eyes by a salient onset distractor and the disengagement of the eyes from the change. The small bars at the bottom of each panel represent the initial distractor color (half green, half gray/red indicates that the distractor changed earlier than when bars are fully green). In all panels, error bars represent within-subjects $95 \%$ confidence intervals (see Bakeman and McArthur 1996)

distractor (i.e. how long the eyes dwell on the distractor before they can move on toward the target) are driven by the same underlying control modes (bottom-up vs. topdown). As was previously demonstrated with the oculomotor capture paradigm (Ludwig and Gilchrist 2002; 2003a; Mulckhuyse et al. 2008; Becker et al. 2009), the current findings confirm that a distractor that has the same color as the target triggers more capture than a distractor that has the same color as the placeholder elements. Also, as was previously shown, a distractor that has the same color as the target produces longer dwell times than a distractor that does not look like the target (Mulckhuyse et al. 2008; Becker et al. 2009). Even though these previous findings seem to suggest that the color of the distractor determines both capture and disengagement in the same way, the current data indicate that the color information may affect capture and disengagement differently:

In the no change condition, we compared the effects of three distractor types: a target-colored distractor (gray; pop-out among the surrounding placeholders), a distractor presented in the placeholder color (red; no pop-out) and a distractor presented in a novel color (green; pop-out). Capture by both pop-out distractors (target and novel color) was stronger than capture by the distractor that did not popout (placeholder color). Importantly, capture with pop-out distractors was the same regardless of whether a novel color or the target color was used. If a strong top-down task-set had influenced capture effects, stronger capture should have occurred with distractors that resembled the target because observers were looking for such a stimulus. As this was not the case, we conclude that whether the distractor looked like the target had no effect on the magnitude of capture; the only thing that mattered was the distractor's bottom-up saliency.

In contrast to the findings regarding capture, dwell times were very much determined by the extent to which the 
distractor looked like the target. Only when the distractor had the same color as the target, observers fixated the distractor relatively long as evidenced by the prolonged dwell times (around $120 \mathrm{~ms}$ ). All distractors that did not resemble the target (i.e. red distractors, green distractors or green distractors that changed to red; see Figs. $2 b, 3 b$ ) produced equally short dwell times of about $80-90 \mathrm{~ms}$. In a similar vein, Born and Kerzel (2009) have shown that a distractor presented at fixation delays saccade initiation more when it is of the same color as the saccade target. This may be interpreted as difficulty to disengage the eyes from the distractor. Importantly, when the color of the target was made irrelevant in their study, the similarity effect was no longer present, demonstrating that it is top-down in nature. Importantly, dwell times in the current study also show no influence of bottom-up saliency: dwell times on the novel color pop-out distractor were as short as dwell times on a distractor that did not pop-out.

Taken together, our results reflect a dissociation between the control of capture and disengagement: on the same trials that showed capture to be influenced by bottom-up saliency, disengagement was heavily influenced by topdown target-distractor similarity effects.

In terms of the competition process between target- and distractor-related activity in the oculomotor system, our finding of a dissociation between the control of capture and disengagement suggests that the time it takes to disengage from a distractor does not depend on its initial power to drive a capture saccade. Apparently, dwell times on the distractor are not the result of residual distractor activity from the previous competition process that has to be overcome to launch a subsequent eye movement. In other words, the amount of residual activity that lingers in the system does not seem to depend on the initial strength of the signal that decided the first competition process (i.e. capture). Instead, after winning the competition, the distractor signal may be maintained and further modulated in a top-down manner, presumably through information from higher-level cognitive areas. Maintenance of the signal that wins the competition could be useful for saccadic error monitoring processes to quickly decide on the subsequent competition that determines whether a corrective saccade has to be initiated. If target-related activity has won the competition, maintenance of the target signal (which will be remapped onto fixation after the saccade) may help preventing a subsequent saccade (Findlay and Walker 1999). If distractorrelated activity has won the competition and the distractor signal is maintained, dwell times reflect that for an element that has the same color as the target, it will take much longer to reject it as a non-target than when it has a different color.

The initial bottom-up saliency advantage (pop-out) of the target color compared to the placeholder color distrac- tors may also partly explain target-distractor similarity effects in capture found in other studies that were attributed to top-down modulations (Ludwig and Gilchrist 2002; Mulckhuyse et al. 2008). However, our findings stand in direct conflict to previous reports of large differences in capture for distractors presented in the target color and distractors in non-target colors, even when the distractor popped out among the placeholder elements (Ludwig and Gilchrist 2003a, Experiment 2; Becker et al. 2009). A close inspection of the data reveals that saccade latencies for capture saccades were much longer in these studies than in the present experiment. They report latencies around $200 \mathrm{~ms}$ or well above, a range that more closely matched the latencies of our target-directed eye movements. In contrast, our capture saccades were initiated about $150 \mathrm{~ms}$ after target onset. The short latencies can be explained by the strong emphasis on speed in our setting: the gap period before target display onset, the use of onset distractors and our explicit speed instruction promoted fast saccade initiation. Moreover, trials in which participants' response took longer than $350 \mathrm{~ms}$ were marked with a "too slow" feedback message and were excluded from analysis (note, however, that only $1.5 \%$ of trials were excluded due to slow responses).Van Zoest and Donk have repeatedly demonstrated that saccades with short latencies are more strongly driven by bottom-up factors and that the influence of top-down control increases with saccade latency (van Zoest et al. 2004; Donk and van Zoest 2008; van Zoest and Donk 2008). In other words, bottom-up oculomotor capture by salient onsets may be reduced for saccades with longer latencies. The discrepancies between previous results (Ludwig and Gilchrist 2003a, Experiment 2; Becker et al. 2009) and the current experiment may be reconciled by assuming that oculomotor capture can be driven by bottom-up or top-down information, depending on the overall speed of the saccadic response. In contrast, dwell times in all studies reflected strong topdown target-distractor similarity effects, regardless of the overall speed of the initial response.

Given that top-down information reaches the oculomotor system with a certain delay, one reason for the dissociation between capture and disengagement in our experiment may be that capture and disengagement operate in different time windows. As revealed by our distractor color change manipulations, capture was largely based on the color that the distractor carried at its onset. If the distractor was presented in the novel color at its onset but changed after a very short delay of 30-40 ms to a color that matched either the target or the color of the placeholder elements, the effect of the color change on capture was very limited. Capture was only slightly less pronounced when the distractor changed to the placeholder color compared to when it changed to the target color. In contrast, capture was much smaller when the distractor appeared in the placeholder 
color compared to when it appeared in the target color (i.e., no change conditions). In other words, the capture pattern for pop-out distractors in a novel color that changed to either target or placeholder color was largely as if the distractor had stayed in the novel pop-out color throughout the trial. Apparently, a color change $30-40 \mathrm{~ms}$ after its onset is too late to strongly influence capture.

In contrast, looking at dwell times, a change at the short delay still produced a large difference in the time to disengage the eye from the distractor that was not reduced compared to the no change condition. In other words, for dwell times, the pattern of results in the short delay condition was rather as if the distractor had never been presented in the novel color at its onset. When the color after the change matched the color of the target, relatively long dwell times were observed; when it matched the color of the placeholder elements, relatively short dwell times were found. The finding that the color change affected capture only weakly and dwell times more strongly suggests that capture did depend on the earliest information reaching the oculomotor system, while information for dwell time was accumulated either during an extended time window from distractor onset, or during a later time period than for capture. Accordingly, during such an extended or shifted integration interval, slow top-down information may start to influence the signals in the oculomotor system (van Zoest et al. 2004; Donk and van Zoest 2008; van Zoest and Donk 2008). Further, an extended or shifted integration interval for disengagement may not be surprising, given that disengagement necessarily occurs after capture.

However, we also found that when the change in distractor color occurred only a bit later in time (at 60-80 ms after distractor onset), even dwell times were no longer affected by the change. That is, dwell times were as if the distractor had not changed color at all. Thus, even though the integration interval for disengagement may have been extended or shifted compared to capture, it still did close surprisingly early. When aligning data to response onset, we found no effects of a distractor change up to $100 \mathrm{~ms}$ before the capture saccade and more than $200 \mathrm{~ms}$ before the corrective saccade was launched. Our results complement previous studies that showed that a saccade-contingent switch in target location during the capture saccade toward a distractor did not influence the subsequent corrective saccade (McPeek et al. 2000; Godijn and Theeuwes 2002): on most trials, the subsequent corrective saccade went to the location that the target occupied before the switch. In terms of saccade programming, the findings corroborate the assumption that the saccade toward the distractor and the subsequent corrective saccade toward the target (including saccadic error monitoring processes determining whether that second saccade is necessary and how fast the eyes can disengage) are partly prepared in parallel (Becker and
Jürgens 1979; Theeuwes et al. 1999; Trappenberg et al. 2001; Godijn and Theeuwes 2002; McPeek and Keller 2002; Walker and McSorley 2006; Murthy et al. 2007, 2009). Only with parallel programming, it is feasible that the dwell time on the distractor, which is essentially a latency component of the second saccade, can only be influenced in the first $60 \mathrm{~ms}$ after target display onset (i.e. well before the initiation of the first response), but not later on. In other words, information from the endpoint of the capture saccade influenced error monitoring processes related to the subsequent competition process for the corrective saccade even before the first saccade had been launched. Critically, dwell time differences between target color and placeholder color distractors already found in previous studies (Mulckhuyse et al. 2008; Becker et al. 2009) apparently do not stem from a feedback signal issued only after the first saccade has landed on the distractor (Murthy et al. 2007). In fact, the lack of difference in our long delay conditions indicate that such a feedback signal did not have any impact at all in our experiment. Note that previous studies on parallel programming of saccades only focused on target-related activity that is evoked before and maintained during the capture saccade to explain short dwell times on the distractor (Becker and Jürgens 1979; Theeuwes et al. 1999; Trappenberg et al. 2001; Godijn and Theeuwes 2002; McPeek and Keller 2002; Walker and McSorley 2006; Murthy et al. 2007, 2009). Thus, the current study complements these earlier findings by showing that early distractor-related signals evoked before the capture saccade also influence dwell times, whereas feedback signals evoked after capture do not.

A phenomenon that may contribute to the fact that late changes neither affect capture nor dwell times is saccadic suppression. Saccadic suppression refers to the finding that visual perception is strongly impaired around the time of saccades. This loss of visual sensitivity begins already $75 \mathrm{~ms}$ before saccade initiation is strongest at saccade onset and lasts until $50 \mathrm{~ms}$ after saccade offset (Diamond et al. 2000). Therefore, any change during the suppression phase of the first distractor-directed saccade may go unnoticed. However, the mechanism seems to selectively inhibit magnocellular signals. For signals conveyed through the parvocellular system, like the color changes used in our study, no suppression is found (Burr et al. 1994). Moreover, even though saccadic suppression may result in participants missing the distractor change, it cannot explain why the external feedback signal coming from the now fixated distractor after the capture saccade does not influence dwell times.

An interesting question is how our findings may relate to covert attentional mechanisms. In the visual search literature, it is still under debate whether the capture of covert attention is under top-down (Folk et al. 1992, 2009; Leber 
and Egeth 2006) or bottom-up control (Theeuwes 1994, 2004, 2010; Schreij et al. 2008). Proponents of the latter view argue that the specific reaction time pattern found when varying the similarity between a cue and a subsequent target that has been attributed to top-down influences on capture may be explained by disengagement processes of covert attention (Theeuwes 1994; Theeuwes et al. 2000; Belopolsky et al. 2010). Irrespective of whether the capture of covert attention may or may not be influenced by topdown control, the strong top-down effects for oculomotor disengagement in the current study suggest that the ease of disengagement of covert attention may also contribute to a large part to top-down effects found in manual reaction times. Frameworks that explain reaction time differences between target-similar and target-dissimilar cues relying solely on the capture component (Folk et al. 1992, 1994) may therefore neglect a vital mechanism of attentional control.

In sum, our experiment showed that when using salient onset distractors that produce short-latency capture saccades, oculomotor capture and disengagement can be dissociated: capture is primarily stimulus driven and strongly dependent on the earliest signals that reach the oculomotor system. In contrast, oculomotor disengagement is under top-down control and dependent on an extended or shifted integration window compared to capture. Nevertheless, beginning only $60-80 \mathrm{~ms}$ after target onset, disengagement was unaffected by distractor changes, which may be explained by parallel programming of saccades. This indicates that it is not the feedback signal from the currently fixated distractor that determines how long the eyes will rest on it; rather, dwell times depend on distractor information that is gathered before the capture saccade is initiated.

Acknowledgments We like to thank Isabel Dombrowe for her help in setting up the experiments. SB and DK were supported by the Swiss National Foundation PDFM1-114417 and a travel grant for SB by the Fondation Ernst \& Lucie Schmidheiny.

Open Access This article is distributed under the terms of the Creative Commons Attribution Noncommercial License which permits any noncommercial use, distribution, and reproduction in any medium, provided the original author(s) and source are credited.

\section{References}

Bakeman R, McArthur D (1996) Picturing repeated measures: comments on Loftus, Morrison, and others. Behav Res Methods 28:584-589

Becker W, Jürgens R (1979) An analysis of the saccadic system by means of double step stimuli. Vision Res 19:967-983

Becker SI, Ansorge U, Horstmann G (2009) Can intertrial priming account for the similarity effect in visual search? Vision Res 49:1738-1756. doi:10.1016/j.visres.2009.04.001
Belopolsky AV, Schreij D, Theeuwes J (2010) What is top-down about contingent capture? Atten Percept Psychophys 72:326-341. doi:10.3758/APP.72.2.326

Born S, Kerzel D (2009) Congruency effects in the remote distractor paradigm: evidence for top-down modulation. J Vis 9:1-13. doi: $10.1167 / 9.9 .3$

Burr DC, Morrone MC, Ross J (1994) Selective suppression of the magnocellular visual pathway during saccadic eye movements. Nature 371:511-513. doi:10.1038/371511a0

Diamond MR, Ross J, Morrone MC (2000) Extraretinal control of saccadic suppression. J Neurosci 20:3449-3455

Donk M, van Zoest W (2008) Effects of salience are short-lived. Psychol Sci 19:733-739. doi:10.1111/j.1467-9280.2008.02149.x

Findlay JM, Walker R (1999) A model of saccade generation based on parallel processing and competitive inhibition. Behav Brain Sci 22:661-674; discussion 674-721

Folk CL, Remington RW, Johnston JC (1992) Involuntary covert orienting is contingent on attentional control settings. J Exp Psychol Hum Percept Perform 18:1030-1044

Folk CL, Remington RW, Wright JH (1994) The structure of attentional control: contingent attentional capture by apparent motion, abrupt onset, and color. J Exp Psychol Hum Percept Perform 20:317-329

Folk CL, Remington RW, Wu SC (2009) Additivity of abrupt onset effects supports nonspatial distraction, not the capture of spatial attention. Atten Percept Psychophys 71:308-313. doi:10.3758/ APP.71.2.308

Godijn R, Theeuwes J (2002) Programming of endogenous and exogenous saccades: evidence for a competitive integration model. J Exp Psychol Hum Percept Perform 28:1039-1054

Irwin DE, Colcombe AM, Kramer AF, Hahn S (2000) Attentional and oculomotor capture by onset, luminance and color singletons. Vision Res 40:1443-1458

Jonides J (1981) Voluntary versus automatic control over the mind's eye's movement. In: Long JB, Baddeley AD (eds) Attention \& performance IX. Erlbaum, Hillsdale, NJ, pp 187-203

Kingstone A, Klein RM (1993) Visual offsets facilitate saccadic latency: does predisengagement of visuospatial attention mediate this gap effect? J Exp Psychol Hum Percept Perform 19:1251-1265

Leber AB, Egeth HE (2006) It's under control: top-down search strategies can override attentional capture. Psychon Bull Rev 13:132-138

Ludwig CJ, Gilchrist ID (2002) Stimulus-driven and goal-driven control over visual selection. J Exp Psychol Hum Percept Perform 28:902-912

Ludwig CJ, Gilchrist ID (2003a) Goal-driven modulation of oculomotor capture. Percept Psychophys 65:1243-1251

Ludwig CJ, Gilchrist ID (2003b) Target similarity affects saccade curvature away from irrelevant onsets. Exp Brain Res 152:60-69. doi:10.1007/s00221-003-1520-7

Ludwig CJ, Ranson A, Gilchrist ID (2008) Oculomotor capture by transient events: a comparison of abrupt onsets, offsets, motion, and flicker. J Vis 8:11 11-16 doi:10.1167/8.14.11

McPeek RM, Keller EL (2002) Superior colliculus activity related to concurrent processing of saccade goals in a visual search task. J Neurophysiol 87:1805-1815. doi:10.1152/jn.00501.2001

McPeek RM, Skavenski AA, Nakayama K (2000) Concurrent processing of saccades in visual search. Vision Res 40:2499-2516

McSorley E, Haggard P, Walker R (2006) Time course of oculomotor inhibition revealed by saccade trajectory modulation. J Neurophysiol 96:1420-1424. doi:10.1152/jn.00315.2006

Mulckhuyse M, van Zoest W, Theeuwes J (2008) Capture of the eyes by relevant and irrelevant onsets. Exp Brain Res 186:225-235. doi:10.1007/s00221-007-1226-3

Mulckhuyse M, Van der Stigchel S, Theeuwes J (2009) Early and late modulation of saccade deviations by target distractor similarity. J Neurophysiol 102:1451-1458. doi:10.1152/jn.00068.2009 
Murthy A, Ray S, Shorter SM, Priddy EG, Schall JD, Thompson KG (2007) Frontal eye field contributions to rapid corrective saccades. J Neurophysiol 97:1457-1469. doi:10.1152/jn.00433. 2006

Murthy A, Ray S, Shorter SM, Schall JD, Thompson KG (2009) Neural control of visual search by frontal eye field: effects of unexpected target displacement on visual selection and saccade preparation. J Neurophysiol 101:2485-2506. doi:10.1152/ jn.90824.2008

Reuter-Lorenz PA, Hughes HC, Fendrich R (1991) The reduction of saccadic latency by prior offset of the fixation point: an analysis of the gap effect. Percept Psychophys 49:167-175

Saslow MG (1967) Effects of components of displacement-step stimuli upon latency for saccadic eye movement. J Opt Soc Am 57:10241029

Schreij D, Owens C, Theeuwes J (2008) Abrupt onsets capture attention independent of top-down control settings. Percept Psychophys 70:208-218

Theeuwes J (1994) Stimulus-driven capture and attentional set: selective search for color and visual abrupt onsets. J Exp Psychol Hum Percept Perform 20:799-806

Theeuwes J (2004) Top-down search strategies cannot override attentional capture. Psychon Bull Rev 11:65-70

Theeuwes J (2010) Top-down and bottom-up control of visual selection. Acta Psychol 135:77-99. doi:10.1016/j.actpsy.2010.02.006

Theeuwes J, Kramer AF, Hahn S, Irwin DE (1998) Our eyes do not always go where we want them to go: Capture of the eyes by new objects. Psychol Sci 9:379-385

Theeuwes J, Kramer AF, Hahn S, Irwin DE, Zelinsky GJ (1999) Influence of attentional capture on oculomotor control. J Exp Psychol Hum Percept Perform 25:1595-1608
Theeuwes J, Atchley P, Kramer AF (2000) On the time course of topdown and bottom-up control of visual attention. In: Monsell S, Driver J (eds) Attention and performance XVIII. MIT Press, Cambridge, pp 105-124

Theeuwes J, De Vries GJ, Godijn R (2003) Attentional and oculomotor capture with static singletons. Percept Psychophys 65:735-746

Todd JT, Gelder PV (1979) Implications of a transient-sustained dichotomy for the measurement of human performance. J Exp Psychol Hum Percept Perform 5:625-638

Trappenberg TP, Dorris MC, Munoz DP, Klein RM (2001) A model of saccade initiation based on the competitive integration of exogenous and endogenous signals in the superior colliculus. J Cogn Neurosci 13:256-271

van Zoest W, Donk M (2008) Goal-driven modulation as a function of time in saccadic target selection. Q J Exp Psychol 61:1553-1572. doi:10.1080/17470210701595555

van Zoest W, Donk M, Theeuwes J (2004) The role of stimulus-driven and goal-driven control in saccadic visual selection. J Exp Psychol Hum Percept Perform 30:746-759

Walker R, McSorley E (2006) The parallel programming of voluntary and reflexive saccades. Vision Res 46:2082-2093. doi:10.1016/ j.visres.2005.12.009

Wu SC, Remington RW (2003) Characteristics of covert and overt visual orienting: Evidence from attentional and oculomotor capture. J Exp Psychol Hum Percept Perform 29:1050-1067. doi:10.1037/ 0096-1523.29.5.1050

Yantis S (1993) Stimulus-driven attentional capture and attentional control settings. J Exp Psychol Hum Percept Perform 19:676-681

Yantis S, Jonides J (1984) Abrupt visual onsets and selective attention: evidence from visual search. J Exp Psychol Hum Percept Perform 10:601-621 